\title{
Experimental demonstration of surface phonon focusing in anisotropic media using laser induced acoustic pulses
}

\author{
Al.A. Kolomenskii, A.A. Masnev, V.G. Mikhalevich and H.A. Schuessler* \\ General Physics Institute, Moscow 117942, Russia \\ * Department of Physics, Texas A\&M University, College Station, TX 77843-4242, U.S.A.
}

\begin{abstract}
The concentration of the acoustic field along preferred directions in different anisotropic single crystals, as well as in a layered medium consisting of a single crystal covered with an amorphous film was studied.
\end{abstract}

\section{INTRODUCTION}

The concentration of the acoustic energy flux due to the crystal anisotropy is usually referred to as phonon focusing. This effect was first observed with ballistic phonons excited by a heat source in a crystal cooled to liquid helium temperatures [1]. However, this focusing effect is a general property of wave propagation in any anisotropic medium. Recently it was also observed at ultrasonic frequencies in a silicon single crystal at room temperature using laser generated bulk [2] as well as surface [3] acoustic waves.

In this paper we present results on the phonon focusing effect with surface acoustic waves (SAW) excited by different pulsed lasers in crystalline Si and GaAs samples and for a layered medium. The layered medium consisted of an amorphous hydrogenated silicon film which had been deposited on a silicon wafer.

\section{THEORY}

The concentration of the acoustic energy flux from a point source is determined [1] by a focusing factor $A$, which for $S A W$ has the form $A \approx 1 /(1+\beta)$, where $\beta=\left(d^{2} c / d \theta^{2}\right) / c$. Here $c(\theta)$ is the dependence of a SAW phase velocity on the angle $\theta$ of the wave vector. $\theta$ is related to the angle $\varphi$ of the observation direction according to $\varphi=\theta+\tan ^{-1}[(\mathrm{dc} / \mathrm{d} \theta) / \mathrm{c}]$. For the directions with $0<1+\beta<<1$, which is realizable for negative values of $\beta$, an increase of the acoustic field occurs and is in the following referred to as a regular maximum.

In crystal cuts with sufficiently strong anisotropy the condition $\beta=-1$ is realized along several distinct directions. In such a direction the focusing factor becomes infinite and a singular maximum arises [4]. These latter directions correspond to the caustics of the acoustic field. A geometrical optics approximation is insufficient for their description. The 


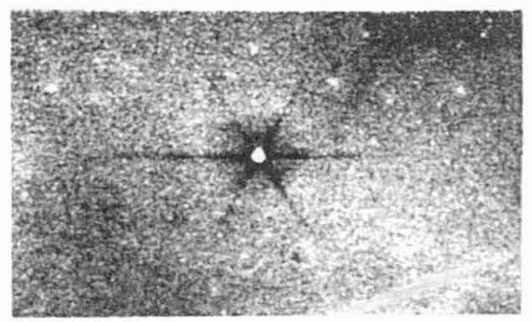

(a)

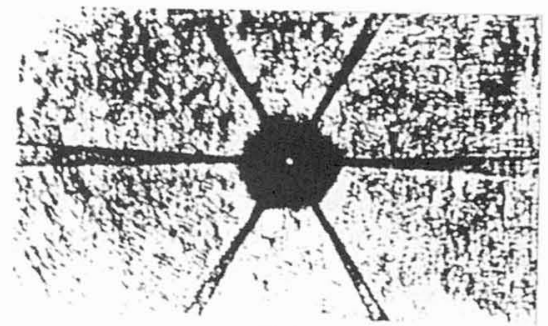

(b)

Fig. 1. Focusing patterns of SAW. (a) for a silicon substrate in the (111) plane covered with a $1 \mu \mathrm{m}$ film of amorphous hydrogenated silicon. (b) for a bare GaAs substrate in the (111) plane.

appearance of a caustic corresponds to the formation of a cusp on the wavefront of the acoustic wave. Therefore singular maxima appear as closely separated pairs.

\section{EXPERIMENTAL PROCEDURE}

In the experiments we used two different setups. The first arrangement used a pulsed nitrogen laser $(\lambda=337 \mathrm{~nm}, \tau=10 \mathrm{nsec}, \mathrm{E}=10 \mathrm{~mJ})$ operated in the multimode regime. A microscope objective was used to produce a spot on a sample surface with the radius $\mathrm{a}=100$ $\mu \mathrm{m}$. In the second setup a Q-switched Nd-YAG laser $(\lambda=1.06 \mu \mathrm{m}, \tau=10 \mathrm{nsec}, \mathrm{E}=10 \mathrm{~mJ})$ operated in single transverse mode produced after focusing a much smaller spot on the sample surface with the radius $\mathrm{a}=7 \mu \mathrm{m}$. In both cases optical breakdown was induced by the laser pulse at the surface and generated the acoustic wave.

\section{RESULTS}

The SAW focusing patterns were registered with the dust visualization technique [3] employing removal of $1-2 \mu \mathrm{m}$ size $\mathrm{A}_{2} \mathrm{O}_{3}$ particles which were deposited on the surface before laser action. After laser action they are partially removed by the passing SAW.

We examined different single crystals. In Fig. 1(a) the focusing pattern for the (111) plane of Si reflects the threefold symmetry with respect to the axis normal to the (111) plane. The focusing directions correspond in this case to regular maxima. Fig. 1(b) depicts pairs of singular maxima for the (111)-plane of a GaAs single crystal which has a stronger anistropy than $\mathrm{Si}$.

A special feature of the result presented in Fig. 1 is that in this case the silicon wafer was covered with a one micron thick film of amorphous hydrogenated silicon. Nevertheless the observed pattern does not differ from that obtained for a bare silicon single crystal [3]. This is due to the fact that the penetration depth of the acoustic field is on the order of the acoustic wavelength, which in our case was about $100 \mu \mathrm{m}$ and was much larger than the thickness of 
the covering film. It is therefore possible to use phonon focusing of SAW to probe the anisotropy and even determine the crystalline orientation of substrates covered with layered materials.

This work is supported by a Teledyne Research Assistance Grant, DOE, and the Center for Energy and Mineral Resources of Texas A\&M University.

\section{References}

[1] G. A. Nortrop and J. P. Wolfe. In: Nonequilibrium Phonon Dynamics. Ed. by W. E. Bron (Plenum, New York, 1985).

[2] A. G. Every and Wolfgang Sachse, Phys. Rev. B44 (1991) 6689.

[3] Al. A. Kolomenskii and A. A. Maznev, JETP Lett. 53 (1991) 423.

[4] V. V. Novikov and L. A. Chernozatonskii, Sov. Phys. Solid State 28 (1986) 233. 